\title{
Random load fluctuations and collapse probability of a power system operating near codimension 1 saddle-node bifurcation
}

\author{
Dmitry Podolsky and Konstantin Turitsyn, Member, IEEE
}

\begin{abstract}
For a power system operating in the vicinity of the power transfer limit of its transmission system, effect of stochastic fluctuations of power loads can become critical as a sufficiently strong such fluctuation may activate voltage instability and lead to a large scale collapse of the system. Considering the effect of these stochastic fluctuations near a codimension 1 saddle-node bifurcation, we explicitly calculate the autocorrelation function of the state vector and show how its behavior explains the phenomenon of critical slowing-down often observed for power systems on the threshold of blackout. We also estimate the collapse probability/mean clearing time for the power system and construct a new indicator function signaling the proximity to a large scale collapse. The new indicator function is easy to estimate in real time using PMU data feeds as well as SCADA information about fluctuations of power load on the nodes of the power grid. We discuss control strategies leading to the minimization of the collapse probability.
\end{abstract}

Index Terms-Blackout prevention, emergency control, phasor measurements, power system stability, voltage stability, wide-area measurements and control.

\section{INTRODUCTION}

I $\mathrm{T}$ is well known that small stochastic fluctuations of power load, although usually negligible in the vicinity of a stable operating point, may potentially lead to a large scale cascading failure if the power system operates close to a saddle-node bifurcation point [1], [2]. Early detection and mitigation of such failures is a problem of utmost importance in contemporary world of constantly increasing power demand, where power grids often operate in a precritical regime.

Statistical properties of aggregated power load, their influence on static and dynamic characteristics of power systems remain the subject of extensive studies for the last three decades, see [1] for the review. A very considerable attention has been given to stability analysis of power networks based on Lyapunov theory of dynamical systems, as it was recognized early that the proximity of an operating point to saddle-node and/or Hopf bifurcations of power systems signals about approach to an instability of the base state [3]. In particular, saddle-node bifurcations were associated to the phenomenon of voltage collapse as well as loss of synchronism [4]. Correspondingly, a multitude of stability criteria based on the estimation of global Lyapunov functions of power systems have been introduced (see for example classical papers [5], [6], [7]), many corresponding indicator functions being already

D. Podolsky and K. Turitsyn are with the Department of Mechanical Engineering, Massachusetts Institute of Technology, Cambridge, MA, 02139. E-mails: podolsky@mit.edu.turitsyn@mit.edu used in industry for preventive control and dynamic security assessment of power grids [1]. Despite its popularity, energy function analysis of power systems is not entirely free of drawbacks: (a) typically, a knowledge of the values of system variables on all nodes of the network is needed in order to estimate the global Lyapunov function in a given operating regime, and (b) even if such tremendous amount of data is available in real time, the estimation process has a high computational cost, which becomes especially critical in the proximity of a large scale collapse.

Assuming in the present work that the operating point of the power system under consideration is close to a saddle-node bifurcation, we extend the standard approach to Lyapunov stability analysis of power systems, taking into account that for a typical power grid without any specific structural symmetries the center manifold is one-dimensional, i.e., saddlenode bifurcation has codimension 1 . This observation allows us to explicitly calculate the autocorrelation function of system variables in the operating regime near the bifurcation point and find an approximate expression for the mean clearing time/probability of a large scale failure of the power system. Using the latter, we construct a new indicator function of proximity to the voltage collapse/loss of synchronism, which is significantly easier to estimate in real time than the global Lyapunov function, especially if the system operator receives real time PMU data as well as SCADA information about fluctuations of power demand on individual nodes.

This manuscript is organized as follows. In Sec. III we review the structure-preserving model used in the present paper to describe dynamic behavior of system variables in the vicinity of the saddle-node bifurcation point. The autocorrelation function of system variables is explicitly calculated in the Sec. III. while a new estimate for the mean clearing time is given in the Sec. IV] In the Sec. V] we perform the validation of our model by checking the derived formulae against numerical simulations of the IEEE 39 bus (New England) power system described in [8]. Finally, the Sec. VI is devoted to discussion of control strategies for minimizing the collapse probability and conclusions.

\section{THE MODEL}

To describe dynamics of system variables in the vicinity of a saddle node bifurcation point, we use the system of coupled 
swing equations on $(P, V)$ nodes of the grid

$$
\frac{H_{i}}{\pi f_{0}} \frac{d^{2} \theta_{i}}{d t^{2}}+\alpha_{i} \frac{d \theta_{i}}{d t}=\sum_{j \sim i} \mathcal{Y}_{i j} V_{i} V_{j} \sin \left(\theta_{i}-\theta_{j}-\gamma_{i j}\right)+P_{m, i}
$$

and power flow equations on $(P, Q)$ nodes

$$
\begin{aligned}
& P_{0, i}+\alpha_{p, i} \dot{\theta}_{i}+\beta_{p, i} V_{i}+T_{p, i} \dot{V}_{i}=\sum_{j \sim i} \mathcal{Y}_{i j} V_{i} V_{j} \sin \left(\theta_{i}-\theta_{j}-\gamma_{i j}\right), \\
& Q_{0, i}+\alpha_{q, i} \dot{\theta}_{i}+\beta_{q, i} V_{i}+T_{q, i} \dot{V}_{i}=\sum_{j \sim i} \mathcal{Y}_{i j} V_{i} V_{j} \cos \left(\theta_{i}-\theta_{j}-\gamma_{i j}\right),
\end{aligned}
$$

where as usual $\theta_{i}$ is a voltage phase on a bus $i, H_{i}$ is an inertia constant for a generator on the node $i, \alpha_{i}$ denote frequency controls on the $(P, V)$ nodes with generators, $V_{i}$ is a voltage magnitude on a bus $i$ (for the $(P, V)$ nodes, $V_{i}=E_{i}$ ). Real and reactive power loads on $(P, Q)$ nodes are generally (weakly changing) functions of frequency $\dot{\theta}_{i}$, voltage $V_{i}$ and voltage change rate $\dot{V}_{i}$. If only dynamics of system variables at the vicinity of a stable operating point is to be considered, it is sufficient to keep first leading orders in the Taylor expansions of real and reactive power loads in powers of their arguments. This corresponds to the structure preserving model of [9] extended to the case of load dependence on the voltage change rate $\dot{V}_{i}$.

The loads have a fixed power factor $k_{i}$. They fluctuate with time, and fluctuations of loads on different nodes of the grid are statistically independent. We assume that their correlation properties are Gaussian:

$$
\left\langle\delta P_{i}(t) \delta P_{j}\left(t^{\prime}\right)\right\rangle=B_{i}\left(t-t^{\prime}\right) \delta_{i j}
$$

One simple example of the function $B_{i}$ is exponential $B_{i}(t-$ $\left.t^{\prime}\right)=A_{i} \exp \left(-\left|t-t^{\prime}\right| / \tau_{i}\right)$, where the characteristic time scale $\tau$ is the decay time of temporal correlations of power load fluctuations.

Since the load power factor is fixed, fluctuations of reactive loads are related to (4) as

$$
\left\langle\delta Q_{i}(t) \delta Q_{j}\left(t^{\prime}\right)\right\rangle=\frac{k_{i}^{2}-1}{k_{i}^{2}} B_{i}\left(t-t^{\prime}\right) \delta_{i j},
$$

so that only fluctuations of $\delta P_{i}$ are needed to be considered.

Note that (4) describes the autocorrelation function of $a$ stationary process. The property of stationarity (or translational invariance in time) $t \rightarrow t+\delta t, t^{\prime} \rightarrow t^{\prime}+\delta t$ is only approximate, as behavior of loads and their random fluctuations features natural cycles (for example, day/night). However, we are only interested to study a short time scale (tens of seconds and minutes) behavior of the state vector, when (4) is perfectly applicable.

After solving the power flow equations, finding the base state and linearizing equations (1), (2), (3) about the stable operating point, one finds the matrix stochastic differential equations (SDE)

$$
\mathcal{M} \ddot{x}+\mathcal{D} \dot{x}+\mathcal{K} x=\mathcal{A} x=\delta P .
$$

Here $x$ is the system state vector including voltage phases and magnitudes, the matrix $\mathcal{M}$ describes inertial properties of generators connected to the grid, the matrix $\mathcal{D}-$ primary frequency controls on $(P, V)$ nodes as well as frequency and $\dot{V}$ dependence of power loads on $(P, Q)$ nodes, and finally $\mathcal{K}$ is the power flow Jacobian encoding all static properties of the power system. The system (5) of SDE will be the main subject of our study.

\section{Calculation of Autocorrelation FUnCtion}

Generally, the autocorrelation function of the system vector $x$ can be found as

$$
\left\langle x(t) x^{T}\left(t^{\prime}\right)\right\rangle=\operatorname{Re} \int \frac{d \omega}{2 \pi} e^{-j \omega\left(t-t^{\prime}\right)}\left\langle x(\omega) x^{\dagger}(\omega)\right\rangle,
$$

where

$$
\left\langle x(\omega) x^{T}(-\omega)\right\rangle=\mathcal{A}^{-1}(\omega) \mathcal{B}(\omega)\left(\mathcal{A}^{\dagger}(\omega)\right)^{-1}
$$

and the Fourier-transformed system matrix is

$$
\mathcal{A}(\omega)=-\mathcal{M} \omega^{2}+j \mathcal{D} \omega+\mathcal{K} .
$$

The correlation properties of fluctuating loads are given by the matrix

$$
\mathcal{B}(\omega)=\left\langle\delta P(\omega) \delta P^{\dagger}(\omega)\right\rangle=B(\omega) \mathbf{1}
$$

The value of the integral (6) is determined by the singularities of the integrand in the complex $\omega$ plane, which in particular include zeros of $\operatorname{det} \mathcal{A}(\omega)$ and $\operatorname{det} \mathcal{A}(-\omega)$, as well as the singularities of $\mathcal{B}(\omega)$. In the regime of interest, dynamics of system variables is largely dictated by very slow modes (see below). Power loads fluctuate rapidly compared to the time scales of this slow dynamics, their correlation in time described by (4) becomes negligible, and one can simply write $\mathcal{B}(\omega)=\mathcal{B} \cdot \mathbf{1}$, where $B_{i j}=\int_{0}^{+\infty} d t B_{i j}(t)$. For the case $B_{i j}(t)=A_{i} \exp \left(-t / \tau_{i}\right) \delta_{i j}$ considered in the previous Section, one expect that the time scales $\tau_{i}$ are the shortest in the system, and one effectively has $B_{i j}=A_{i} / \tau_{i} \delta_{i j}$.

Naturally, the singularity closest to the real $\omega$ axis determines behavior of the autocorrelation function (6) at late times. To identify it, we note that at a saddle-node bifurcation point several eigenvalues of the power flow Jacobian $\mathcal{K}$ vanish. There exists only one such vanishing eigenvalue $\epsilon \rightarrow 0$ if the center manifold of the power system is one-dimensional [12]. Performing the eigenvalue decomposition of the inverse power flow Jacobian

$$
\mathcal{K}^{-1}=\sum_{i} b_{i} \Lambda_{i}^{-1} a_{i}^{T}
$$

where $\Lambda_{i}$ are eigenvalues of $\mathcal{K}$ and $a_{i}$ and $b_{i}$ are corresponding left and right eigenvectors, we see that if the operating point of the power system is close to the saddle-node bifurcation point, $\mathcal{K}^{-1}$ is approximately given by

$$
\mathcal{K}^{-1}=\frac{1}{\epsilon} b a^{T}+\ldots
$$

where $\epsilon$ is the eigenvalue of $\mathcal{K}$ vanishing at the bifurcation point. Dots denote the contribution of all the other eigenvalues of $\mathcal{K}$, which is at most of the order $\mathcal{O}(1)$ in powers of $\epsilon$ (although for large systems it can be numerically large). We are particularly interested in the situation when the 2-norm $\left\|\frac{1}{\epsilon} b a-\mathcal{K}^{-1}\right\|_{2} \ll\left\|\mathcal{K}^{-1}\right\|_{2}$ and the asymptotic representation (7) holds well. 
In the case under consideration, the leading singularity of the integrand in $\sqrt{6}$ coincides with a zero of $\operatorname{det} \mathcal{A}(\omega)$ (or $\operatorname{det} \mathcal{A}(-\omega)$ depending on the sign of the time difference $\left.t-t^{\prime}\right)$. Such singularity is a simple pole by assumption that the center manifold of the power system is one-dimensional. Solving the equation $\operatorname{det} \mathcal{A}(\omega)=\operatorname{det}\left(-\mathcal{M} \omega^{2}+i \mathcal{D} \omega+\mathcal{K}\right)=0$ by means of perturbation theory in the small parameter $\epsilon$, one finds that the mode determining behavior of the autocorrelation function at late times is given by

$$
\omega_{0} \approx-\frac{j}{\operatorname{Tr}\left(\mathcal{D} \mathcal{K}^{-1}\right)}=-\frac{j \epsilon}{a^{T} \mathcal{D} b}+\mathcal{O}\left(\epsilon^{2}\right) .
$$

The frequency of the leading mode is purely imaginary 1

Estimating the integral (6) in the vicinity of this leading singularity, one finally finds (to the leading order in $\epsilon$ )

$$
\left\langle x(0) x^{T}(t)\right\rangle=\frac{b\left(a^{T} \mathcal{B} a\right) b^{T}}{2 \epsilon a^{T} \mathcal{D} b} \exp \left(-\frac{\epsilon t}{a^{T} \mathcal{D} b}\right) .
$$

Note that in this operating regime near the codimension 1 saddle-node bifurcation point dynamics of system variables $x(t)$ is directly related to static characteristics of the power system such as the lowest eigenvalue $\epsilon$ of the power flow Jacobian.

As follows from (9), the right eigenvector $b$ of the power flow Jacobian $\mathcal{K}$ determines the preferred direction in the phase space of a power system near a codimension 1 saddlenode bifurcation [12], [11]. In turn, the left eigenvector $a$ shows fluctuations of the loads on which nodes mostly determine stochastic dynamics of the state vector: the nodes $i$ with larger components $a_{i}$ are the ones, where fluctuations of the power loads $\delta P_{i}, \delta Q_{i}$ influence the dynamics of the state vector stronger.

As the operating point of the power system approaches the power transfer limit and the lowest eigenvalue $\epsilon$ of the power flow Jacobian gets smaller, the rate of decay of the autocorrelation function (9) decreases quickly. This observation is known as the phenomenon of critical slowing-down observed during large scale failures in power grids [13], [14].

Finally, we would also like to emphasize that fluctuations of the leading mode, the component of the system vector $x$ along $b$ direction, become strongly amplified as $\epsilon$ decreases, since the amplitude of the leading mode near the bifurcation is proportional to $\epsilon^{-1 / 2}$. One of the main goals of system control in this regime is to decrease the amplitude of the leading mode by adjusting the values of the matrix element $a^{T} \mathcal{D} b$ (and, if possible, $a^{T} \mathcal{B} a$ ). This can be done using primary frequency control on $(P, V)$ nodes as well as utilizing resources of load following on $(P, Q)$ nodes as we discuss below.

\section{MEAN CLEARING TIME AND PROBABILITY OF VOLTAGE COLLAPSE}

Taking the results of the previous Section into account, it is also possible to explicitly calculate the mean clearing time for an operating point close $e^{2}$ to the bifurcation point.

\footnotetext{
${ }^{1}$ An oscillating contribution disappears from the mode for sufficiently small $\epsilon<\left(a^{T} \mathcal{D} b\right)^{2} / 4 a^{T} \mathcal{M} b$.

${ }^{2}$ But not too close as will become clear from the subsequent discussion.
}

Namely, considering a scalar reduced system variable $z(t)$ defined according to

$$
z(t)=b^{T} x(t)
$$

one finds the following equation of motion for it:

$$
a^{T} \mathcal{M} b \cdot \ddot{z}+a^{T} \mathcal{D} b \cdot \dot{z}+\epsilon z+a^{T} \Gamma b b \cdot z^{2}+\ldots=a^{T} \delta P,
$$

where the matrix $\Gamma_{i j k}=\frac{\partial \mathcal{K}_{i j}}{\partial \theta_{k}}$ is the first derivative of the power flow Jacobian w.r.t. the system variables and ... denote higher derivatives of $\mathcal{K}$. The equation (11) is a scalar SDE describing the process of activation of an over-damped unharmonic oscillator. Therefore, one can simply apply the classical result by Kramers [18] to calculate the probability of collapse/the mean clearing time:

$$
t_{\mathrm{mct}} \approx \frac{2 \pi a^{T} \mathcal{D} b}{\epsilon} \exp \left(\frac{\epsilon^{3} a^{T} \mathcal{D} b}{3\left(a^{T} \Gamma b b\right)^{2}\left(a^{T} \mathcal{B} a\right)}\right)
$$

Estimates similar to this one were previously found using Lyuapunov stability analysis of power systems (see for example [6], [7]). Yet, the expression [12] is different from these well-known results in one important respect: the mean clearing time (12) depends only on a small number of parameters of the power system, unlike the global Lyuapunov function which is a functional of dynamical characteristics of the power system. This is so because the number of degrees of freedom of a power system gets enormously reduced in the vicinity of bifurcation, and all the information about the power system in the vicinity of the bifurcation point is aggregated through a small number of quantities. These quantities (such as matrix elements $a^{T} \mathcal{D} b$ and $a^{T} \mathcal{B} a$ or the lowest eigenvalue $\epsilon$ of the power flow Jacobian) entering (12) can be straightforwardly estimated from the static state analysis using the measurements of the correlation function $\left\langle x(0) x^{T}(t)\right\rangle$ of the state vector (containing the information about the vector $b$, the matrix element $a^{T} \mathcal{D} b$ and $\epsilon$ ) and local SCADA data of power consumption on individual nodes of the grid (which encode information about the matrix $\mathcal{B}$ ) and therefore do not require any a priori knowledge of the power system structure. Such measurements will make it easier to estimate the mean clearing time for the power system in real time using the expression (12).

We also emphasize that the result (12) is strictly speaking applicable only to the case of power systems with onedimensional center manifold, and an estimate similar to (12) would be impossible to make if the dimension of center manifold of the power system is 2 or larger. What is necessary for the derivation of 12 is the equilibration law known in physics as the principle of detailed balance, and it does not apply for system matrices of generic power grids. However, because of tremendous reduction of the total number of degrees of freedom in the vicinity of a codimension 1 bifurcation point, the power system becomes effectively one-dimensional (there is only one relevant degree of freedom), and the principle of detailed balance is always valid for one-dimensional systems.

\section{NUMERICAL SIMULATION OF IEEE 39 POWER SYSTEM}

We shall now perform the verification of the model described in the Section II comparing the expression (9) for the 
correlation function with results of numerical simulations of the IEEE 39 (New England) power system. The strategy is as follows. First, the saddle-node bifurcation point is localized using continuation power flow procedure [15] implemented in PSAT Toolbox for Matlab [16], then it is identified more precisely using MATPOWER 4.1 library for Matlab [17]. An operating point close to the bifurcation is chosen, static power flow equations - solved using MATPOWER, the power flow Jacobian - calculated, and finally stochastic differential equations of the Section $\mathrm{II}$ are solved using the implicit Euler algorithm. The number $N_{r}$ of realizations of the random load fluctuations is 1000 (we consider only a stationary regime where averaging the system variables $x(t)$ over realizations of the statistical ensemble (4) of random loads is expected to be equivalent to averaging of $x(t)$ over time).

The load parameter $\lambda=2.12$ is chosen (if $\lambda=1$ corresponds to the operating point described in [8]). This corresponds to a $0.9 \%$ displacement in terms of the generated real power as compared to the critical regime $3^{3}$ As usual, the bus 31 is a slack bus. The state vector includes voltage phases on the nodes 1-30, 32-39 and voltage magnitudes on the nodes 1-29. The same $\mathcal{M}$ matrix chosen as presented in [8], with the base frequency $f_{0}=60 \mathrm{~Hz}$. The $\mathcal{D}$ matrix is diagonal with components $d=1$ p.u. corresponding to voltage phases on $(P, Q)$ buses, $d=0.1$ p.u. corresponding to voltage magnitudes on $(P, Q)$ buses and $d=10$ p.u. - to phases on $(P, V)$ buses. The loads are allowed to fluctuate only on the buses 3, 10 and 21, with the same characteristic amplitudes of fluctuations $\sqrt{B}=0.1$ p.u. For our choice of $\lambda$ the smallest eigenvalue of the power flow Jacobian $\mathcal{K}$ is $\epsilon \approx 0.1811$ p.u, with the ratio of 2 -norms $\left\|\mathcal{K}^{-1}-\epsilon^{-1} b a\right\|_{2} /\left\|\mathcal{K}^{-1}\right\|_{2} \approx 0.0717$. Therefore, the expansion in powers of $\epsilon$ is feasible, albeit $\epsilon$ is not too small by itself (only smallness of dimensionless quantities dictates where the perturbation theory is applicable).

The numerical results for the autocorrelation function $\left\langle z(t) z\left(t^{\prime}\right)\right\rangle$ of the reduced system variable $z(t)=b^{T} x(t)$ as well as the scalar autocorrelation function $\left\langle x^{T}(t) x\left(t^{\prime}\right)\right\rangle$ are presented on the Fig. 11 The full correlation function of the system vector $\left\langle x^{T}(t) x\left(t^{\prime}\right)\right\rangle$ clearly follows the one of reduced variable $\left\langle z(t) z\left(t^{\prime}\right)\right\rangle$ except the initial short interval of time, where contributions from sub-leading modes into the autocorrelation function are not small. This confirms our expectation that fluctuations of system variables orthogonal to the direction of the vector $b$ become suppressed in the vicinity of the saddle-node bifurcation. Note that for any given finite number of realizations $N_{r}$ the autocorrelation function $\left\langle x^{T}(t) x\left(t^{\prime}\right)\right\rangle$ fluctuates stronger than $\left\langle z(t) z\left(t^{\prime}\right)\right\rangle$ because it also accounts for fluctuations of the system vector orthogonal to the vector $b$, although in the limit of infinite number of realizations both correlation functions coincide with each other.

For our choice of parameters the value of the amplitude of the correlation function $\left\langle x^{T}(t) x\left(t^{\prime}\right)\right\rangle$ is $a^{T} \mathcal{B} a / 2 \epsilon a^{T} \mathcal{D} b \approx$ $5.96 \cdot 10^{-4}$, while the best fit value recovered from 1 is $5.26 \cdot 10^{-4}$. The autocorrelation decay time $\tau=a^{T} \mathcal{D} b / \epsilon \approx$

\footnotetext{
${ }^{3}$ The total generated real power at $\lambda_{\text {crit }} \approx 2.13569843$ is approximately $13.67 \mathrm{GW}$.
}

$17.3 \mathrm{sec}$ also coincides well with the best fit value $\tau \approx 17.0$ sec and an integral estimate for the average correlation time $\int_{0}^{t_{f}} d t\left\langle x^{T}(t) x\left(t^{\prime}\right)\right\rangle /\left\langle x^{T}(t) x(t)\right\rangle \approx 18.1 \mathrm{sec}$.

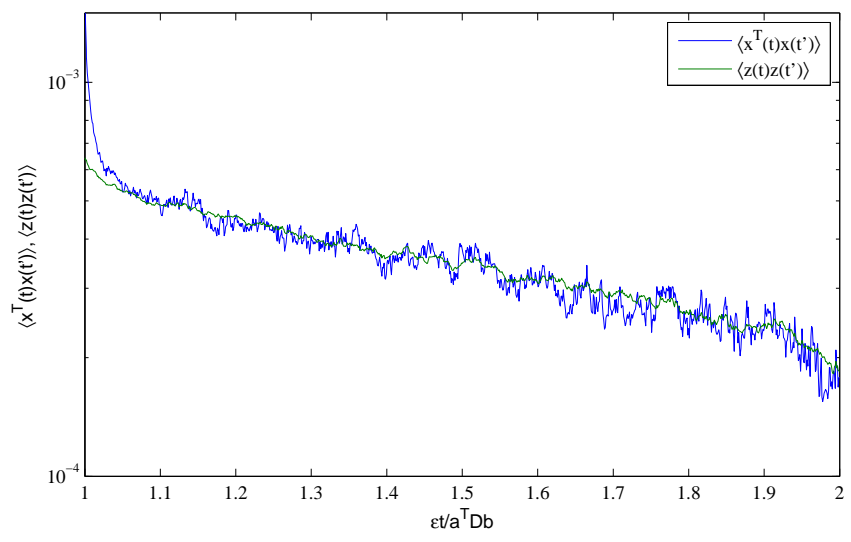

Figure 1. Relation between the logarithms of the autocorrelation function $\left\langle z(t) z\left(t^{\prime}\right)\right\rangle$ of the reduced system variable $b^{T} x(t)$ (red) and the full autocorrelation function $\left\langle x^{T}(t) x\left(t^{\prime}\right)\right\rangle$ (blue). The initial moment of time $t$ is chosen to be $t=a^{T} \mathcal{D} b / \epsilon$.

\section{DISCUSSION AND PATH FORWARD}

In the present paper we discuss a general power grid without any particular structural symmetries operating near the power transfer limit of its transmission system. A saddle-node bifurcation present in the phase space of such power system typically has codimension 1, which implies that dynamics of state variables near such bifurcation point is essentially determined by a single degree of freedom which we denote as reduced system variable. We consider how stochastic fluctuations of power loads exactly influence this dynamics, explicitly calculating (a) the autocorrelation function of the state vector (9) and (b) the probability of a large scale failure of the power system in the vicinity of the saddle-node bifurcation point/the mean clearing time for the system (12). Although such an operating regime was extensively studied in literature for the last 30 years (see [1] and references therein), both results (a) and (b) are new to our knowledge. Also, using (a) we are able to quantify the phenomenon of critical slowing-down recently discovered in power grids operating on the threshold of a large scale cascading failure [13], [14].

Using the expression for the mean clearing time (12), one can introduce a new simple indicator function (13) signaling proximity of a power system to a large scale failure. Indeed, as follows from the expression (12), collapse probability is exponentially small when the expression

$$
I_{c}=\frac{\epsilon^{3} a^{T} \mathcal{D} b}{\left(a^{T} \Gamma b b\right)^{2} a^{T} \mathcal{B} a} \gg 1
$$

and vise versa. Even if the operating point of the power system under consideration is close to the power transfer limit (i.e., $\epsilon$ nearly vanishes), the mean clearing time could still be exponentially large when the matrix element $a^{T} \mathcal{D} b$ is large and/or the matrix element $a^{T} \mathcal{B} a-$ small. This in turn implies that the probability of a large scale failure is low. 
As follows from (13), a strategy for a system operator to keep the power system close to the power transfer limit of its transmission system while minimizing the probability of a large scale collapse would be to maximally utilize resources of load following [21] (effectively reducing the matrix element $a^{T} \mathcal{B} a$ ) and/or adjust, if it is possible, parameters of the primary frequency control on $(P, V)$ nodes (increasing the value of the matrix element $\left.a^{T} \mathcal{D} b\right)$. Note that $a^{T} \mathcal{D} b$ cannot be increased indefinitely: such increase for example would imply a growth of the correlation time $\tau=a^{T} \mathcal{D} b / \epsilon$ leading in turn to possible issues related to interference between primary and secondary frequency controls.

There exist several reasons why the operating regime of a power system discussed in the paper seems rather attractive despite the fact that the operating point is located near the threshold of system instability. First of all, in such a regime, the power grid and its transmission system are utilized with the best effectiveness possible: the throughput of the transmission system is maximal, nearly all energy which the grid is capable to produce is consumed (assuming the minimal power losses in the transmission system). Therefore, such operating regime of the grid is optimal from the economical point of view given the system remains under control and the operating costs of control systems are not too high.

Second, synchronism of the power system actually holds rather well in the regime under consideration. Indeed, the degree of synchronism is determined by the correlation function of the operating frequency $\left\langle\delta \omega_{i}(t) \delta \omega_{j}\left(t^{\prime}\right)\right\rangle$, which for the nodes $i$ with large representation in the vector $b$ is given to the leading order in $\epsilon$ by

$$
\left\langle\delta \omega_{i}(t) \delta \omega_{j}\left(t^{\prime}\right)\right\rangle \approx \frac{\epsilon b_{i}\left(a^{T} \mathcal{B} a\right) b_{j}}{\left(a^{T} \mathcal{D} b\right)^{3}} \exp \left(-\frac{\epsilon\left|t-t^{\prime}\right|}{a^{T} \mathcal{D} b}\right) .
$$

Control countermeasures necessary to maximize the value of the indicator function (13) and minimize the collapse probability are the ones which also maximize the value of the matrix element $a^{T} \mathcal{D} b$ (and/or minimize $a^{T} \mathcal{B} a$ ) and therefore decrease the amplitude of the correlation function (14).

Finally, since in the regime under consideration dynamics of the power system is essentially described by a single degree of freedom, the complicated problem of state recovery can be effectively solved by using real time data feeds from synchrophasor measurement units as well as a much larger (and much slower acquired) volume of SCADA data about fluctuating aggregated power loads. The same applies to the problem of system identification [22]: as we have discussed, measurements of the autocorrelation function $\left\langle x(0) x^{T}(t)\right\rangle$ using PMU data feeds directly provide information about the matrix elements $a^{T} \mathcal{D} b$ and $a^{T} \mathcal{B} a$, the lowest eigenvalue of the power flow Jacobian, corresponding left and right eigenvectors $a$ and $b$. Therefore, such measurements can potentially allow for the approximate recovery of the system matrix in the vicinity of the bifurcation point or at least its part responsible for the dynamics of the leading mode of the state vector. We shall discuss this observation more extensively elsewhere.

\section{ACKNOWLEDGMENTS}

This work was partially supported by NSF award ECCS1128437 and MIT/SkTech seed funding grant.

\section{REFERENCES}

[1] C. Canizares, "Voltage Stability Assessment: Concepts, Practices and Tools", IEEE/FES Power System Stability Subcommittee, Technical Report, 2002.

[2] G. Andersson, P. Donalek, R. Farmer, N. Hatziargyriou, I. Kamwa, P. Kundur, N. Martins, J. Paserba, P. Pourbeik, J. Sanchez-Gasca, R. Schulz, A. Stankovic, C. Taylor, and V. Vittal, "Causes of the 2003 Major Grid Blackouts in North America and Europe, and Recommended Means to Improve System Dynamic Performance," IEEE Transactions on Power Systems, vol. 20, no. 4, pp. 1922-1928, Nov. 2005.

[3] H. Kwatny, R. Fischl, and C. Nwankpa, "Local bifurcation in power systems: theory, computation, and application," Proceedings of the IEEE, vol. 83, no. 11, pp. 1456-1483, 1995.

[4] C. W. Taylor, Power system voltage stability. McGraw- Hill, 1994.

[5] C. De Marco and A. Bergen, "A security measure for random load disturbances in nonlinear power system models," IEEE Transactions on Circuits and Systems, vol. 34, no. 12, pp. 1546-1557, Dec. 1987.

[6] C. De Marco and T. Overbye, "An energy based security measure for assessing vulnerability to voltage collapse," IEEE Transactions on Power Systems, vol. 5, no. 2, pp. 419-427, May 1990.

[7] C. Nwankpa and R. Hassan, "A stochastic based voltage collapse indicator," IEEE Transactions on Power Systems, vol. 8, no. 3, pp. 1187-1194, 1993.

[8] M.A. Pai, Energy function analysis for power system stability. Kluwer Academic Publishers, 1989.

[9] A. Bergen and D. Hill, "A Structure Preserving Model for Power System Stability Analysis," IEEE Transactions on Power Apparatus and Systems, vol. PAS-100, no. 1, pp. 25-35, Jan. 1981.

[10] I. Dobson and H.-D. Chiang, "Towards a theory of voltage collapse in electric power systems," Systems \& Control Letters, vol. 13, no. 3, pp. 253-262, Sep. 1989.

[11] C. Canizares, "On bifurcations, voltage collapse and load modeling," IEEE Transactions on Power Systems, vol. 10, no. 1, pp. 512-522, 1995.

[12] I. Dobson, "Observations on the geometry of saddle node bifurcation and voltage collapse in electrical power systems," IEEE Transactions on Circuits and Systems I: Fundamental Theory and Applications, vol. 39, no. 3, pp. 240-243, Mar. 1992.

[13] P. Hines, E. Cotilla-Sanchez, and S. Blumsack, "Topological Models and Critical Slowing down: Two Approaches to Power System Blackout Risk Analysis," in 2011 44th Hawaii International Conference on System Sciences. IEEE, Jan. 2011, pp. 1- 10.

[14] E. Cotilla-Sanchez, P.D.H. Hines, C.M. Danforth, "Predicting Critical Transitions from Time Series Synchrophasor Data," submitted, 2012.

[15] V. Ajjarapu and C. Christy, "The continuation power flow: a tool for steady state voltage stability analysis," IEEE Transactions on Power Systems, vol. 7, no. 1, pp. 416-423, 1992.

[16] F. Milano, "An open source power system analysis toolbox," IEEE Transactions on Power Systems, vol. 20, no. 3, pp. 1199-1206, 2005.

[17] R.D. Zimmerman, C.E. Murillo-Sanchez, R.J. Thomas, "MATPOWER: Steady-State Operations, Planning, and Analysis Tools for Power Systems Research and Education," IEEE Transactions on Power Systems, vol.26, no.1, pp.12-19, Feb. 2011.

[18] H. Kramers, "Brownian motion in a field of force and the diffusion model of chemical reactions," Physica, vol. 7, no. 4, pp. 284-304, Apr. 1940.

[19] J. Machowski, J. Bialek, and D. J. Bumby, Power System Dynamics: Stability and Control. John Wiley \& Sons, 2011.

[20] S. Redner, A guide to first-passage processes. Cambridge University Press, 2001.

[21] E. Hirst, B. Kirby, Electric-power ancillary services. Oak Ridge National Laboratory, 1996.

[22] L. Ljung, System Identification: Theory for the User. Prentice Hall, 1998. 